\title{
QUANTIFYING CLOSED LOOP PERFORMANCE BASED ON ON-LINE PERFORMANCE INDICES
}

\author{
M. Farenzena and J. O. Trierweiler ${ }^{\#}$ \\ Group of $\underline{\text { Integration, }}$ Modeling, $\underline{\text { Simulation, }}$ Control and Optimization of $\underline{\text { Processes }}$ (GIMSCOP) \\ Department of Chemical Engineering, Federal University of Rio Grande do Sul (UFRGS) \\ Rua Luiz Englert, s/n CEP: 90.040-040 - Porto Alegre - RS - BRAZIL, \\ Fax: +55 5133163277 , Phone: +55 5133164072 \\ E-MAIL: \{farenz,jorge\}@enq.ufrgs.br
}

\begin{abstract}
This article aims to construct an "inference model" (IM) that assesses the closed loop performance and robustness for SISO controllers, with no need of intrusive tests (i.e. set-point changes or open-loop tests). The input variables are indexes that can be easily calculated on-line (minimal variance, perceptual error, standard deviation, among others). The IM is generated for a large set of plants and tuning parameters. The possible inputs for the IM are 9 standard assessment measurements (e.g., Harris Index, standard deviation, etc) on-line available, commonly present in commercial tools. The target for IM is the closed loop open loop rise time ratio $\left(\mathrm{Rt}_{\mathrm{R}}\right)$, Gain Margin (GM), and normalized (ISE). These values are obtained by intrusive tests. Four different classes of inferential models (i.e., Neural Networks, Neuro Fuzzy, PLS, and QPLS) are compared. The best results are obtained by Neural Network IM. The results obtained show that the methodology is very promising. Copyright $@ 2006$ IFAC
\end{abstract}

Keywords: Performance Monitoring, Neural Network, Performance Assessment.

\section{INTRODUCTION}

Process control increases the plant performance by the reduction of the variability of the key variables. After the variability reduction, the process can achieve a new operating point nearer the restrictions, where the profit is higher (Marlin, 1995).

Even if the actual controller performance is good, some factors can deteriorate the performance during the operation:
- $\quad$ Equipment fouling

- Sensor/actuator problems

- Seasonal influence

- Feed changes

- Operating point changes

Assess on-line the performance of each controller is essential to keep the plant in a profitable operating point. However, quantify the performance of each controller in a typical refinery of petrochemical plant is a difficult task, due to the large amount of loops (usually about 1000 to 2000 loops).

\footnotetext{
\# Author to whom the correspondence should be addressed.
} 


\section{UNIVERSIDADE FEDERAL DO RIO GRANDE DO SUL Seminário do Programa de Pós -Graduação em Engenharia Química Oktober Fórum 2005 - PPGEQ}

The aim of this work is build an inferential model (IM) to the closed loop performance and robustness indexes. The inputs of this IM are the indexes commonly present in commercial tools, described in section 2 .

This paper is organized as follows: section 2 provides an overview about the main performance indexes. Section 3 shows the main limitations of these techniques and in section 4 an inferential model to determine the closed loop performance based on online indexes is introduced. In section 6, different techniques to build the inferential model to predict closed loop performance is shown, which is tested through a case study in section 7 .

\section{ON-LINE INDEXES}

This section shows the most used indexes to assess closed loop performance available in the commercial software. These indexes will be used as the input of the inferential model proposed in this work.

\subsection{Minimal Variance Index}

Harris (1989) proposed an index that assesses the performance of controllers using the minimal variance controller as benchmark Huang and Shah, (1996). The performance index, proposed by Harris $(\eta(d))$ is calculated by the following relation:

$$
\eta(d)=\frac{\sigma_{M V}{ }^{2}}{\sigma_{y}{ }^{2}}
$$

where $\sigma_{M V}{ }^{2}$ is the variance produced by the minimum variance controller and $\sigma_{\mathrm{y}}{ }^{2}$ is the actual loop variance.

The values of $\eta$ always are between 0 and 1 . Increasing values of $\eta$ indicates the performance becomes better. The actual variance is easy to determine in a window of closed loop data. However, the minimal variance for a given control loop is more difficult. It depends both on the plant and the kind of disturbance. Several methodologies to estimate the minimum variance are described in Huang and Shah, (1996).

The main advantage of minimal variance index is only closed loop data is required to assess the performance.

\subsection{Index related to Controlled Variables (CV)}

Another quite simple possibility is to use indexes based on the error of the controlled variable. The most used are:

- Standard deviation of CV (StdCV)

- ISE of CV (ISECV)

- Perceptual error of CV (E\%CV), i.e. the mean absolute error of controlled variable divided by the mean value of $\mathrm{CV}$.

All these indexes are calculated using only closedloop data. No invasive tests are needed.

\subsection{Index related to Manipulated Variables (MV)}

Indexes that quantify the work of the manipulated variable are also used to estimate the performance. The most used are:

- Minimal variance calculated over the manipulated variable ( $\eta \mathrm{MV})$

- Travel of manipulated variable (MV) (TMV)

- $\quad$ Number of inversions of MV(IMV)

\subsection{Commercial Software}

The indexes discussed in the last sections together with other ones are available in almost all commercial software for performance assessment (e.g., ProcessDoctor of Matrikon, PlantTriage of ExperTune, TriPerfeX of TriSolutions, among others). These modern tools give to engineer a huge amount of indexes, which are not so conclusive and easy to interpret. To solve this problem, in section 4 it is proposed a novel approach, but before it is interesting to analyze the main limitations the current methodologies.

\section{LIMITATIONS CONCERNING THE PREVIOUS METHODOLOGIES}

This section shows the limitation of the methodologies available in commercial software to estimate the performance of controllers. The main index is based on the Harris's Index. Despite the fact that this index aims to generate a unique and absolute grade to quantify the performance of the controllers, the estimation of minimal variance is not error free.

The main limitations of conventional on-line index are:

- Only performance is assessed, no information about robustness is provided. 


\section{UNIVERSIDADE FEDERAL DO RIO GRANDE DO SUL \\ Seminário do Programa de Pós -Graduação em Engenharia Química \\ Oktober Fórum 2005 - P PGEQ}

- The scale is not absolute (i.e. there is no guaranty that a loop with $\eta(d)=0.6$ have a better performance than other loop for other process variable with $\eta(d)=0.4$ )

- The spam of the scale is deficient. In some cases, the difference between a poor and a good tuning is very small.

To illustrate these limitations, consider two different systems with the following transfer functions:

$$
G_{1}=\frac{1}{2 s+1} e^{-s} \quad \text { and } \quad G_{2}=\frac{1}{(3 s+1)^{2}} e^{-0.5 s}
$$

Three different PI controllers were designed for each plant, using Frequency Domain Methodology (Trierweiler et al. 2000):

- $\quad$ with the closed loop performance 6 times faster than open loop (6X),

- equal closed and open loop performance (1X),

- closed loop 4 times slower than open loop (0.25X).

The Harris's index calculated for the two plants with the three controllers is shown in Table 1. This simple example clearly shows the scale and resolution problems of Harris's index.

To overcome these limitations, commercial software try to assess the performance based on heuristics that consider the performance indexes and the characteristics of the loop (flow, temperature, pressure, etc.), (Thornhill et al., 1999).

\section{Table 1: Harris indexes for two different plants with three different controllers}

\begin{tabular}{ccc}
\hline Controller & Plant 1 & Plant 2 \\
\hline $6 \mathrm{X}$ & 0.8823 & 0.7338 \\
$1 \mathrm{X}$ & 0.7638 & 0.3306 \\
$0.25 \mathrm{X}$ & 0.5724 & 0.0729 \\
\hline
\end{tabular}

\section{DEVELOPING AN INFERENTIAL MODEL}

The main reason of failure of conventional performance indexes is the absence of a common and absolute target to quantify the performance and robustness. These absolute index are common in the literature, however they are infeasible to determine in a real plant, because invasive tests are necessary.
In this work, a set of representative indexes are determined using a set of plants with different characteristics and several control loop performance measurements calculated for several controller and plant pairs. In these plants, invasive tests are made and the absolute indexes are calculated. After that, using on-line measured indexes, a curve that gives the absolute indexes is fitted, using different techniques. Figure 1 shows a schematic representation of the inferential model proposed in this work.

The main contribution of this work is build an inferential model for performance and robustness indexes that need intrusive tests to be calculated, using only indexes that can be quantified on-line.

The performance and robustness indexes to be inferred are:

- Ratio between closed loop and open loop rise time $\left(\mathrm{Rt}_{\mathrm{R}}\right)$;

- Integral square error (ISE) for a unitary setpoint change and unitary load disturbance normalized by the ISE of a controller with the same performance of open loop;

- Gain margin (GM).

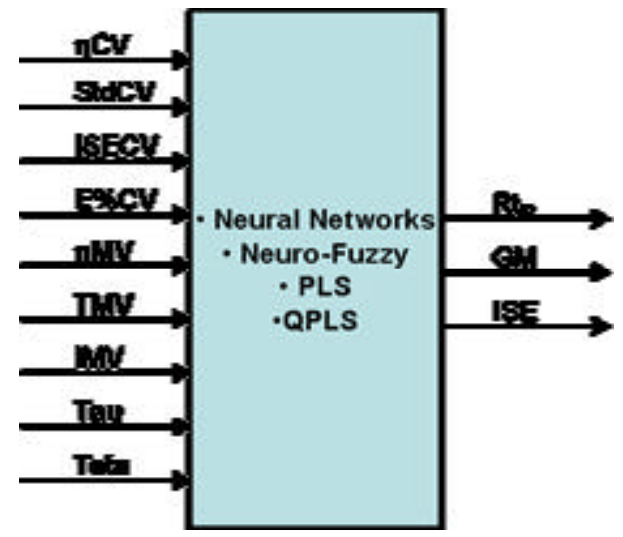

Figure 1: Schematic representation of the inferential model proposed

To develop the inferential model the following four different techniques will be will be tested:

- Neural Networks

- Neuro-Fuzzy (ANFIS)

- PLS

- $\quad$ QPLS 


\section{UNIVERSIDADE FEDERAL DO RIO GRANDE DO SUL Seminário do Programa de Pós -Graduação em Engenharia Química Oktober Fórum 2005 - PPGEQ}

\section{THE PLANTS AND CONTROLLERS}

\subsection{The Plants}

Initially a set of plants to be analyzed are determined, emphasizing different effects: dynamics, model order, RHP zeros, pure time delay, RHP-poles, and integrating processes. These plants are very similar to used by Åström, and Hägglund (1995) to develop the Kappa-Tau PID tuning method.

\subsection{The Controllers}

The controllers are tuned using the Frequency Domain Methodology (Engell and Müller, 1993). For each plant, the desired performance is determined and the methodology gives the parameters for each plant, when the desired performance is achievable. In all cases, the controller used is Proportional-Integral (PI). Table 2 shows the desired performance for the controller, when it is achievable.

The system is affected by white noise and a periodical load disturbance with variable frequency and constant magnitude (unitary). The indexes are calculated in each scenario (each plant with each controller performance) with four different periods for the periodical disturbances $(10,20,30$ and 50 time units).

About $80 \%$ of points are used to train each fit method and $20 \%$ of points are used to test the curves and quantify the correlation among the points. The points of the test set are selected randomly from the total set.

Table 2: Set of desired performances for the controllers

\begin{tabular}{ccc}
\hline $\mathrm{N}$ & $\begin{array}{c}\text { Closed loop/open loop } \\
\text { performance ratio }\end{array}$ & Overshoot $(\%)$ \\
\hline 1 & 10 & 10 \\
2 & 8 & 10 \\
3 & 6 & $20,10,5$ \\
4 & 5 & 10 \\
5 & 4 & $20,10,5$ \\
6 & 2 & 10 \\
7 & 1.5 & 10 \\
8 & 1 & 5 \\
9 & 0.75 & 5 \\
10 & 0.5 & 5 \\
11 & 0.25 & 5 \\
\hline
\end{tabular}

\subsection{Variable selection}

A subset of original variables is selected, using a Genetic Algorithms (GA). The implemented algorithm has the traditional operators (cross-over, reproduction, and mutation), with binary codification (Han and Yang, 2004). A maximum set of 5 variables could be selected. The objective function, to be minimized, is the predictive sum of squares (PRESS) (Qi and Zhang, 2001). The five variables selected by the GA are the same for three developed inferential models (i.e., $\mathrm{Rt}_{\mathrm{R}}$, $\mathrm{GM}$, and ISE):

- Estimated the CV minimal variance

- Process dead time (estimated)

- Process time constant (estimated)

- Standard deviation of CV

- Travel of manipulated variable (MV)

\section{RESULTS}

This section shows the results of the developed inferential models by different techniques the set of plants.

The results will be presented with more details for the ratio between closed loop and open loop rise time.

The target to be achieved is normalized using logarithm, because the most controllers are faster than closed loop, and some are slower. This normalization gives more importance to he faster controllers, and makes the interpolation more representative.

To quantify the quality of each method, the correlation coefficient $\left(\mathrm{R}^{2}\right)$ is calculated, given by: where $\mathrm{R}^{2}$ is the covariance between $\mathrm{y}_{\beta}$ (predicted value) and y (target).

\subsection{Neural Network Inferential Model}

The first approach to build the inferential model was based on neural networks (Hagan, 1995). The neural network used has 2 layers, the first using hyperbolic tangent sigmoid neurons with variable number and the second with one linear neuron. The train method used is Levenberg-Marquardt backpropagation (Hagan, 1995). The first test aims to estimate the ratio between closed loop and open loop rise time. Table 3 shows the performance of several neural networks with different number of neurons in the hidden layer. The results are calculated considering the validation data. 


\section{UNIVERSIDADE FEDERAL DO RIO GRANDE DO SUL \\ Seminário do Programa de Pós -Graduação em Engenharia Química \\ Oktober Fórum 2005 - P PGEQ}

Table 3: Relationship between neural networks performance and number of neurons

\begin{tabular}{cc}
\hline Neurons & $\mathrm{R}^{2}$ for the validation data \\
\hline 10 & 0.91 \\
20 & 0.96 \\
30 & 0.96 \\
40 & 0.96 \\
50 & 0.97 \\
60 & 0.97 \\
\hline
\end{tabular}

The best relationship between prediction quality versus number of neurons is 20. Figure 2 shows the relationship between the predicted and real values for the closed/open loop ratio for each controller.

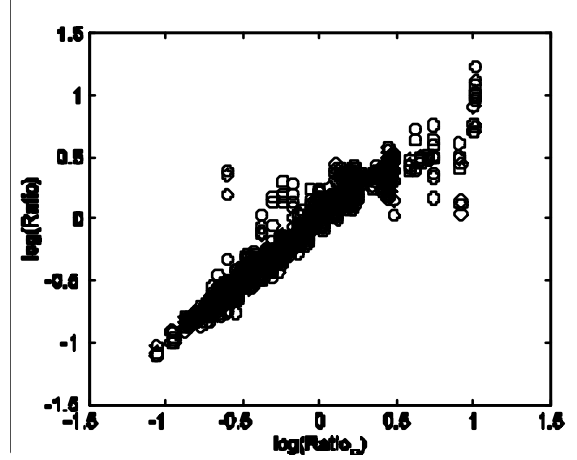

Figure 2: Estimation of $\mathrm{Rt}_{\mathrm{R}}$ rise time using neural networks for the validation data

Table 4: Relationship between neural networks performance for gain-margin (GM) and Integral of Square Error (ISE) with different neural-networks

\begin{tabular}{ccc}
\hline Neurons & $\mathrm{R}^{2}$ for $\mathrm{GM}$ & $\mathrm{R}^{2}$ for ISE \\
\hline 10 & 0.95 & 0.96 \\
20 & 0.96 & 0.98 \\
30 & 0.97 & 0.99 \\
40 & 0.97 & 0.99 \\
50 & 0.98 & 0.99 \\
60 & 0.98 & 0.99 \\
\hline
\end{tabular}

As shown in Figure 2 and Table 3, the prediction of the neural networks is very good. Similar results are also obtained for the gain margin and the integral of square error (ISE) as it is shown in Table 4.

\subsection{Neuro-Fuzzy (ANFIS) Inferential Model}

The second class of inferential model, which has been tested, is Neuro-Fuzzy. The architecture used is ANFIS proposed by Jang (1993). The system has the same five inputs used by the neural networks. The output to be fitted is closed loop and open loop rise time ratio $\left(\mathrm{Rt}_{\mathrm{R}}\right)$. The ANFIS has 2 membership functions, for each input. The architecture of each membership function is S-Functions (Kasabov, 1998). Figure 3 shows the result using neuro-fuzzy approach.

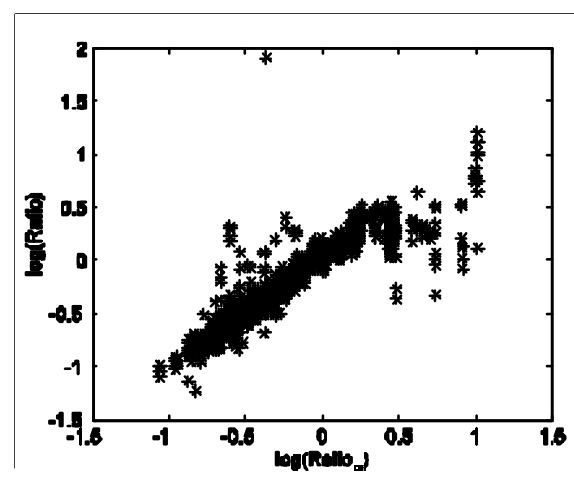

Figure 3: Interpolation of the $\mathrm{Rt}_{\mathrm{R}}$ time using neuro-fuzzy

Figure 4 shows that the predictive capacity of the Neuro-Fuzzy models is less effective than by neural networks. The $\mathrm{R}^{2}$ obtained is also lower (0.91). Similar results are obtained for gain-margin and integral square error (ISE). The correlation factors obtained are 0.89 and 0.96 , respectively.

\subsection{Partial Least Squares (PLS) Inferential Model}

PLS is a very robust technique to interpolate correlated and noise data BAFFI et al. (1999). In this section, the latent variables are defined as linear functions of input variables. All inputs are provided to the algorithm, and different number of latent variables is used, as shown in Table 5:

Table 5: Correlation factor for the inferential models for $\mathrm{Rt}_{\mathrm{R}}$ using linear PLS models

\begin{tabular}{cc}
\hline Latent variable & $\mathrm{R}^{2}$ for the validation data \\
\hline 1 & 0.76 \\
2 & 0.81 \\
3 & 0.81 \\
4 & 0.82 \\
5 & 0.82 \\
6 & 0.82 \\
\hline
\end{tabular}

Figure 4 shows the best result obtained with linear PLS. The correlation index $\left(\mathrm{R}^{2}\right)$ (cf. Table 5) and Figure 4 show that the inferential quality is very poor. Similar results are obtained for GM and ISE, as shown in Table 6. 


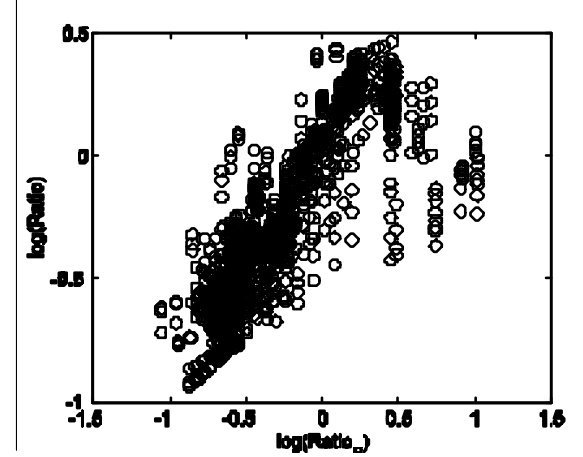

Figure 4: Interpolation of $\mathrm{Rt}_{\mathrm{R}}$ using linear PLS

Table 6: Results obtained by inferential linear PLS models developed for gain-margin (GM) and Integral of Square Error (ISE) with different number of latent variables

\begin{tabular}{ccc}
\hline Latent variable & $\mathrm{R}^{2}$ for $\mathrm{GM}$ & $\mathrm{R}^{2}$ for ISE \\
\hline 1 & 0.74 & 0.73 \\
2 & 0.87 & 0.82 \\
3 & 0.89 & 0.83 \\
4 & 0.90 & 0.84 \\
5 & 0.90 & 0.84 \\
6 & 0.90 & 0.84 \\
\hline
\end{tabular}

6.4 Quadratic Partial Least Squares (QPLS) Inferential Model

QPLS is an alternative to improve the results of PLS models (BAFFI et al., 1999). Table 7 shows the obtained results with QPLS approach.

Table 7: Correlation factor for the inferential models for closed loop and open loop rise time ratio using QPLS models

\begin{tabular}{cc}
\hline Latent variable & $\mathrm{R}^{2}$ \\
\hline 1 & 0.83 \\
2 & 0.84 \\
3 & 0.86 \\
4 & 0.87 \\
5 & 0.88 \\
6 & 0.88 \\
\hline
\end{tabular}

Table 8: Results obtained by inferential QPLS models developed for gain-margin (GM) and Integral of Square Error (ISE) with different number of latent variables

\begin{tabular}{ccc}
\hline Latent variable & GM & ISE \\
\hline 1 & 0.90 & 0.86 \\
2 & 0.90 & 0.88 \\
3 & 0.90 & 0.89 \\
4 & 0.91 & 0.90 \\
5 & 0.91 & 0.91 \\
6 & 0.92 & 0.91 \\
\hline
\end{tabular}

The QPLS models gave a good result, however inferior than neural networks. Similar results are obtained for the inferential models developed for GM and ISE (cf. Table 8).

\section{APPLYING TO THE CASE STUDY}

The example shown in section 3 is used to verify the prediction quality of the neural network inferential model to predict $\mathrm{Rt}_{\mathrm{R}}$. Table 9 compares the results for each case. The true values are in the first column while the corresponding predictions are in the other columns for each plant.

Table 9: Prediction of the best inferential model (IM)

\begin{tabular}{ccc}
\hline \multicolumn{3}{c}{ for two plants } \\
\hline True Values & Plant 1 & Plant 2 \\
\hline $6 \mathrm{X}$ & 5.3 & 5.7 \\
$1 \mathrm{X}$ & 0.87 & 1.3 \\
$0.25 \mathrm{X}$ & 0.37 & 0.24 \\
\hline
\end{tabular}

Table 9 shows that the IM gives representative results for $\mathrm{Rt}_{\mathrm{R}}$. These results are much superior and conclusive than of the obtained by the Harris index. With these results, a control engineer can easily quantify the performance for each controller for the plant.

\section{CONCLUSIONS}

The work presented in this paper built an "inference model" that can really and conclusively quantify closed-loop performance. This novel approach is based on measurements that can be easily assessed on-line, without intrusive tests. The indexes determined are closed loop and open loop rise time ratio, gain margin (GM) and integral square error (ISE).

A set of plants are generated, considering different processes and a set of controllers, with different performances are tuned for each plant. The input indexes are calculated and invasive tests are made to determine the output indexes. The set of input output indexes are fitted using different techniques. The best results are obtained using neural networks, for the three output indexes. Neuro-Fuzzy and QPLS have also good results. Linear PLS gave the worst results. 


\section{UNIVERSIDADE FEDERAL DO RIO GRANDE DO SUL \\ Seminário do Programa de Pós -Graduação em Engenharia Química \\ Oktober Fórum 2005 - PPGEQ}

Based on the results obtained, we can say that the IM proposed can not only assess the performance of industrial controllers, but quantify the real closedloop performance and robustness, with absolute indexes, with no need of intrusive tests.

\section{ACKNOWLEDGMENT}

The authors thank CAPES, PETROBRAS and FINEP for supporting this work.

\section{REFERENCES}

Åström, K. J.; Hägglund, T.; (1995). PID Controllers: Theory, Design, and Tuning. $2^{\mathrm{a}}$ ed. Research Triangle Park: Instrument Society of América.

Baffi, G.; Martin E. B.; Morris A. J.; (1999), NonLinear Projection To Latent Structures Revisited: The Quadratic PLS Algorithm. Computers \& Chemical Engineering, V.23 , 395-411.

Engell, S. E.; Müller, R.; (1993) Multivariable Controller Design by Frequency Response Approximation. Proc. of the 2nd European Control Conference, 3, 1715-1720.

Hagan, M.T.; Demuth, H.B.; Beale, M.; (1995). Neural Networks Desing. PWS Publishing Company.

Han, S. H.; Yang, H.; (2004). Screening Important Design Variables For Building A Usability Model: Genetic Algorithm-Based Partial Least Squares Approach. Industrial Ergonomics, V.33, 159-171.

Harris, T. J.; Seppale, C. T.; Desborough, L. D.; (1999). A review of performance monitoring and assessment techniques for univariate and multivariate control systems. Journal of Process Control, Vol 9, pp. 1-17.

Huang, B.; Shah, S.; (2001) Performance Assessment of Control Loops. Theory \& Applications, Springer, 1999, ISBN 1-85233-6390; DM 139, Journal of Process Control, Volume 11, Issue 4, Pages 441-442.

Jang, J.-S.R. (1993).ANFIS: adaptive-network-based fuzzy inference system.IEEE Transaction on System Man and Cybernet, 23(5), 665-685.

Kasabov, N. K.; (1998). Foundations on Neural Networks, Fuzzy Systems, and Knowledge Engineering. The MIT Press, Cambridge, Massachusetts.

Qi, M; Zhang, G. P.; (2001). An Investigation of
Model Selection Criteria For Neural Network Time Series Forecasting. European Journal of Operational Research V.132, 666-680.

Thornhill, N.F.; Oettinger, M.; Fedenczukc (1999). Refinery-wide control loop performance assessment. Journal of Process Control V.9, 109124

Trierweiler, J. O.; Müller, R.; Engell, S. (2000). Multivariable Low Order Structured-Controller Design by Frequency Response Approximation. Brazilian Journal of Chemical Engineering, 17, n. ${ }^{\circ} 4,793-807$. 